\title{
A Relative Study on Image Segmentation Methods
}

\author{
Mohammed Jajere Adamu ${ }^{1}$, Xuewen Ding ${ }^{2}$ \\ ${ }^{1}$ School of Electronic Engineering, Tianjin University of Technology and Education, 1310, Dagu South, Hexi District, Tianjin, P. R. China \\ ${ }^{2}$ Institute of Image Processing, School of Electronic Engineering, Tianjin University of Technology and Education, \\ 1310, Dagu South, Hexi District, Tianjin, P. R. China
}

\begin{abstract}
Image segmentation is the building block to image analysis and detailed data extraction from them. The problems of digital image segmentation offers various challenges to researchers in this field. This paper tries to explain the principles of some basic methods used to segment an image. This paper discuss the trend of each algorithm with their applications, advantages and disadvantages. This study is helpful for appropriate use of existing segmentation methods and for improving their performance as well as for systematically designing new methods.
\end{abstract}

Keywords: Image, Pixel, Threshold, Segmentation, Algorithm

\section{Introduction}

An image is basically a two dimensional function of spatial, $\mathrm{f}(\mathrm{x}, \mathrm{y})$, and amplitude of this function at a given coordinate gives the intensity value of the image. The image can be expressed as the product of functions of illumination and reflection.

$$
f(x, y)=i(x, y) \cdot r(x, y)
$$

where $i(x, y)$ is the function of intensity and $r(x, y)$ is the function of reflectivity.

In digital image processing, computer algorithm is used to perform image processing which improve the quality of the image by removing noise \& other unwanted pixels and also to obtain more information on the image. Image segmentation is one of the most critical tasks in automatic image analysis. This paper mainly focus on the various methods of image segmentation followed by few algorithms that are widely used. An attempt is made to provide the comparison on these methods by taking test images. MATLAB software was used for image operation in this paper.

\section{Image Segmentation}

Image segmentation is a mid-level processing technique used to analyze the image and can be defined as a processing technique used to classify or cluster an image into several disjoint parts by grouping the pixels to form a region of homogeneity based on the pixel characteristics like gray level, color, texture, intensity and other features. The main purpose of the segmentation process is to get more information in the region of interest in an image which helps in annotation of the object scene [5]. Image segmentation aims at domain-independent partition of the image into a set of visually distinct and homogeneous regions with respect to certain properties [13]. The main goal of segmentation is to clearly differentiate the object and the background in an image. If $\mathrm{R}$ represents an image, then the image segmentation is simply division of $\mathrm{R}$ into sub regions $\mathrm{R}_{1}, \mathrm{R}_{2} \ldots \ldots \mathrm{R}_{\mathrm{n}}$, such that and is governed by following set of rules: a) $R_{i}$ is a connected set, $i=1,2, \ldots . . n$

b) $R_{i} \cap R_{j}=\varnothing$ for all $i$ and $j, i \neq j$

c) $Q\left(R_{i}\right)=$ True for $i=1,2, \ldots n$.

d) $Q\left(R_{i} U R_{j}\right)=$ False for adjoint regions, $R_{i}$ and $R_{j}$

Where $Q\left(R_{k}\right)$ is a logical predicate [19]. The rules described above mentions about continuity, one-to-one relationship, homogeneity and non-repeatability of the pixels after segmentation respectively. There are many knowledge based approaches to segment an image and can be listed as follows:

1) Intensity based methods

2) Discontinuity based methods

3) Similarity based methods

4) Clustering methods

5) Graph based methods

6) Pixon based methods

7) Hybrid methods

In the subsequent sections a brief discussion is made on some of these methods mentioned above in order to understand the principles and extract the attributes from the region of interest.

\section{Related Works}

The segmentation of the image is very useful in medical applications to diagnose the abnormalities in the image [8][6], satellite imaging and in computer vision as well as in Artificial Neural Network. The criteria for segmenting the image is very hard to decide as it varies from image to image and also varies significantly on the modality used to capture the image. There is large amount of literature available to understand and analyze the segmentation techniques [1]. Clustering methods have been used for medical image segmentation in particularly for MR Images of brain and are successful in combining fuzzy $\mathrm{c}$ means and k-means to get novel fuzzy-k means algorithm. Few limitations of the obtained algorithm have been also stated. Hybrid technique for medical image segmentation is suggested in [2] and mainly works on fuzzy-c means and Otsu's method after applying on vector median filter, for segmentation and have tried to prove the 


\section{International Journal of Science and Research (IJSR) \\ ISSN (Online): 2319-7064 \\ Index Copernicus Value (2015): 78.96 | Impact Factor (2015): 6.391}

robustness of their method few kinds of noise have been added to image and have obtained satisfactory results. A new technique for general purpose interactive segmentation of $\mathrm{N}$-dimensional images using graph-cut method has been proposed by Yuri and Jolly [16]. In their proposed method the user marks certain pixels as "object" or "background" to provide hard constraints for segmentation. They claim that their method gives best balance of boundary and region properties compared to other segmentation methods and also that it provides optimal solution for $\mathrm{N}$-dimensional segmentation. The list of related works done in the field of image segmentation is very large and can hardly be mentioned. There are various survey and reviews [8][12][6] done on these methods periodically. The following sections gives brief principle used to segment images.

\section{Intensity Based Segmentation}

One of the simplest approaches to segment an image is based on the intensity levels and is called threshold based approach. Threshold based techniques classifies the image into two classes and works on the postulate that pixels belonging to certain range of intensity values represents one class and the rest of the pixels in the image represents the other class. Thresholding can be implemented either globally or locally. Global thresholding distinguishes object and background pixels by comparing with threshold value chosen and use binary partition to segment the image. The pixels that pass the threshold test are considered as object pixel and are assigned the binary value " 1 " and other pixels are assigned binary value " 0 " and treated as background pixels. The threshold based segmentation techniques are inexpensive, computationally fast and can be used in real time applications with aid of specialized hardware [20].

$$
g(x, y)= \begin{cases}1 & \text { for } \mathrm{i}(\mathrm{x}, \mathrm{y}) \geq \mathrm{t} \\ 0 & \text { for } \mathrm{i}(\mathrm{x}, \mathrm{y})<t\end{cases}
$$

where $\mathrm{g}(\mathrm{x}, \mathrm{y})$ is the output image; $\mathrm{i}(\mathrm{x}, \mathrm{y})$ is the input image and $t$ is the threshold value. Local thresholding is also called adaptive thresholding. In this technique the threshold value varies over the image depending on the local characteristic of the subdivided regions in the image. The algorithm followed for adaptive thresholding can be stated in general as:

1)Divide the image into subimage.

2)Choose a local threshold for subimage considered.

3)Compare the pixels in that subimage and segment the region.

4)Consider all subimages individually and choose corresponding threshold values. Stop segmentation when all the subimages are processed.

In case of global thresholding the threshold value chosen remains the same for the entire image and acts as a cutoff value. In case of local thresholding the image is to be subdivided in to subimages and the threshold is to be chosen depending on the properties of local pixels in that subimage. Threshold value can be modified and are categorized as band thresholding, multi-thresholding and semi-thresholding. Either the global thresholding or local thresholding yield the result depending on the value of threshold chosen. Hence the choice of threshold is crucial and complicated. There are several methods employed for detection of threshold value to name a few mean method, p-tile-thresholding, bimodal histogram, optimal thresholding, multispectral thresholding, edge maximization method [20] of the available techniques for threshold based segmentation, threshold selection based on the histograms suggested by Nobuyuki Otsu in 1979 is most used with minor modifications [15]. Otsu method is optimal for thresholding large objects from the background. This method provides an optimal threshold (or set of thresholds) selected by the discriminant criterion by maximizing the discriminant measure $\eta$ (or the measure of separability of the resultant classes in gray levels) [7]. The other approaches employed to select threshold value are histogram based methods, clustering based methods, mutual information based methods, attribute based methods and local adaptive segmentation based methods. The following results compare these techniques.

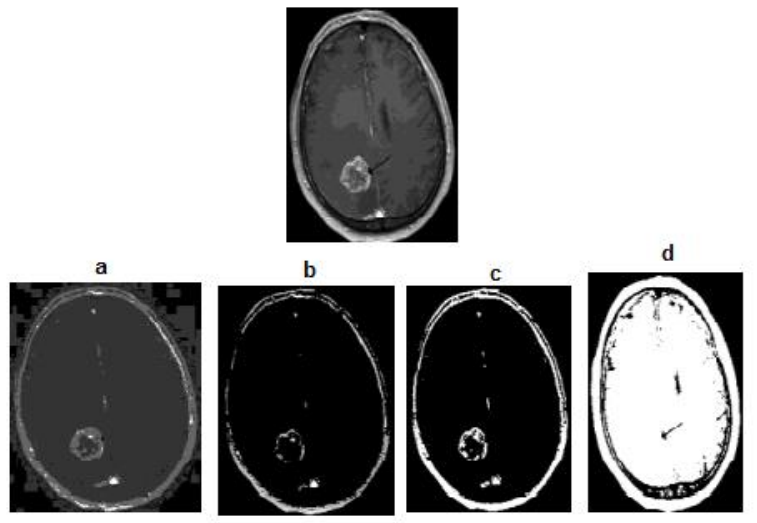

Figure 1: Results showing four different methods:

a. Multi-thresholding

b. Semi-thresholding

c. Band-thresholding

d. Otsu's method

Advantages of threshold based methods:

1) Computationally inexpensive.

2) Fast and simpler to implement.

3) Can work in real-time applications [20].

Disadvantages of threshold based algorithms:

1)Neglects the spatial information of the image.

2)Highly noise sensitive.

3) Selection of threshold value is crucial and often results in over or under segmentation.

4)May lead to pseudo edges or missing edges [14].

\section{Discontinuity Based Methods}

These methods are based on the principle of intensity variations among the pixels. If the image consists two or more objects boundaries exists and hence can be applied to segment the image. The boundaries of the objects lead to formation of edges. The significant abrupt changes in the intensity levels among neighboring pixels

\section{Volume 6 Issue 2, February 2017




\section{International Journal of Science and Research (IJSR) \\ ISSN (Online): 2319-7064 \\ Index Copernicus Value (2015): 78.96 | Impact Factor (2015): 6.391}

in certain direction are termed as edges and results in the discontinuity in the pixels. Edge detection basically involves the following steps: smoothing the image, edge detection and edge localization [3]. A suitable smoothing filter is applied on test image to remove the noise from the image to make it suitable for segmentation. Then the possible edges are grouped together to check for candidature and finally the true edges are found by localizing the edge candidates. There are four different edge types that may be present in the image (a) step-edge (b) ramp edge (c) ridge edge and (d) ramp edge and are shown in the figures correspondingly [11] (a)

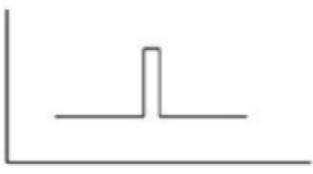

(c)

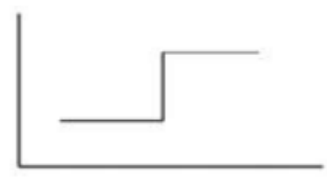

(b)

(d)
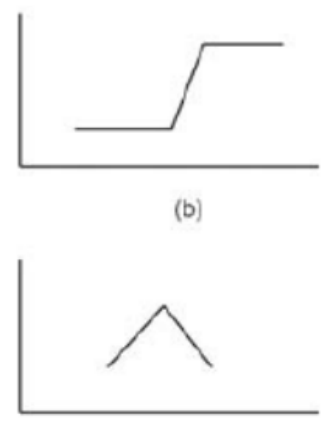

Figure 2: Sub figures a, b, c, and d showing the four different edge types

Edges are usually found by applying masks over the image. The gradient or the zero crossing techniques are employed to find the edges in the given image. The convolution operation between the mask and the image determines the edge set for the image. Edge detection operators can be broadly classified into two categories as: First order derivative operators and Second order derivative operators.

\section{A.First Order Derivative Operators}

There are two methods for first order derivative edge detection. One of the methods is evaluating the gradients generated along two orthogonal directions. The second approach is utilizing a set of discrete edge templates with different orientations. The first derivative operator uses gradient method to find the edges by using the maximum and minimum value of the gradient. The gradient is a measure of change in a function.

$$
\nabla f=G\lfloor f(x, y)\rfloor=\left[\begin{array}{l}
G_{x} \\
G_{y}
\end{array}\right]=\left[\begin{array}{l}
\frac{\partial f}{\partial x} \\
\frac{\partial f}{\partial y}
\end{array}\right]
$$

Direction of gradient is given by

$$
\alpha=\tan ^{-1}\left[\frac{g_{y}}{g_{x}}\right]
$$

$\alpha$ is measured with respect to $\mathrm{x}$ axis.

The operators used in this category are Robert's operator, Prewitt's operator and Sobel's operator.

i. Robert's operator: It is the simplest first derivative operator that can be used to find the edges in the given images. It finds the edges in row and columns separately and are put together to find the resultant edge. The masks used to solve the purpose along $\mathrm{x}$ and $\mathrm{y}$ direction respectively are

\begin{tabular}{|c|c|}
\hline-1 & 0 \\
\hline 0 & 1 \\
\hline
\end{tabular}

\begin{tabular}{|l|l|}
\hline 0 & -1 \\
\hline 1 & 0 \\
\hline
\end{tabular}

ii. Prewitt's operator: This operator uses $3 \times 3$ mask to find the edges. The mask used along $\mathrm{x}$ and $\mathrm{y}$ direction correspondingly are

\begin{tabular}{|c|c|c|}
\hline-1 & -1 & -1 \\
\hline 0 & 0 & 0 \\
\hline 1 & 1 & 1 \\
\hline
\end{tabular}

\begin{tabular}{|l|l|l|}
\hline-1 & 0 & 1 \\
\hline-1 & 0 & 1 \\
\hline-1 & 0 & 1 \\
\hline
\end{tabular}

iii. Sobel's Operator: This is widely used first derivative operator to find edges and is modification of Prewitt's operator by changing the center coefficient to 2 . The sobel operators are given as

\begin{tabular}{|c|c|c|}
\hline-1 & -2 & -1 \\
\hline 0 & 0 & 0 \\
\hline 1 & 2 & 1 \\
\hline
\end{tabular}

\begin{tabular}{|c|c|c|}
\hline-1 & 0 & 1 \\
\hline-2 & 0 & 2 \\
\hline-1 & 0 & 1 \\
\hline
\end{tabular}

iv. The Frei-Chen mask: is another operator used to find the edges in the image and its corresponding masks are given as

\begin{tabular}{|c|c|c|}
\hline 0 & 0 & -1 \\
\hline$\sqrt{2}$ & 0 & $\sqrt{2}$ \\
\hline 0 & 0 & -1 \\
\hline
\end{tabular}

\begin{tabular}{|c|c|c|}
\hline-1 & $\sqrt{2}$ & -1 \\
\hline 0 & 0 & 0 \\
\hline 1 & $\sqrt{2}$ & 1 \\
\hline
\end{tabular}

\section{B.Second Order Derivative Operators}

These operators work on zero crossing detection of the second derivative of the gradient. It detects the local maxima in gradient values and treats them as edges. The Laplacian operator is used with the second derivative operator. The Laplacian operator for any function $f(x, y)$ is given by:

$$
\nabla^{2}=\frac{\partial^{2} \mathrm{f}}{\partial \mathrm{x}^{2}}+\frac{\partial^{2} \mathrm{f}}{\partial \mathrm{y}^{2}}
$$

Where $\frac{\partial^{2} f}{\partial x^{2}}=f(x, y+1)-2 f(x, y)+f(x, y-1)$

$$
\frac{\partial^{2} \mathrm{f}}{\partial \mathrm{y}^{2}}=\mathrm{f}(\mathrm{x}+1, \mathrm{y})-2 \mathrm{f}(\mathrm{x}, \mathrm{y})+\mathrm{f}(\mathrm{x}-1, \mathrm{y})
$$

The frequent used second derivative operators for edge detection are Laplacian of Gaussian (LoG) operator and Canny edge operator.

\section{Volume 6 Issue 2, February 2017




\section{International Journal of Science and Research (IJSR) \\ ISSN (Online): 2319-7064}

Index Copernicus Value (2015): 78.96 | Impact Factor (2015): 6.391

\section{a. Laplacian of Gaussian Operator:}

The Laplacian of an image highlights regions of rapid intensity change. The operator normally takes a single gray level image as input and produces another gray level image as output. The kernels used for approximation of second derivatives Laplacian operations are

\begin{tabular}{|c|c|c|}
\hline 1 & 1 & 1 \\
\hline 1 & -8 & 1 \\
\hline 1 & 1 & 1 \\
\hline
\end{tabular}

\begin{tabular}{|c|c|c|}
\hline-1 & 2 & -1 \\
\hline 2 & -4 & 2 \\
\hline-1 & 2 & -1 \\
\hline
\end{tabular}

\begin{tabular}{|c|c|c|}
\hline 0 & 1 & 0 \\
\hline 1 & -4 & 1 \\
\hline 0 & 1 & 0 \\
\hline
\end{tabular}

\section{b. Canny Edge Operator:}

Canny edge operator is considered as superior edge detection operator among the available operators based on the experimental results as it determines strong and weak edges in the image. Image is first smoothed by using circular two-dimension Gaussian function, computing the gradient of the result and then using the gradient magnitude and direction to approximate edge strength and direction at every point. The gradient magnitude array so obtained consists of undesirable ridges around local maxima and are to be suppressed to get discrete orientations of the edge normal by the process of non-maxima suppression. Then the technique of double thresholding is employed to reduce false fragments. Two thresholds are used to solve the purpose $T_{1}$ and $T_{2}$ where $T_{2} \approx 2 T_{1}$. The following results compare the above said methods to segment image using edge operators.
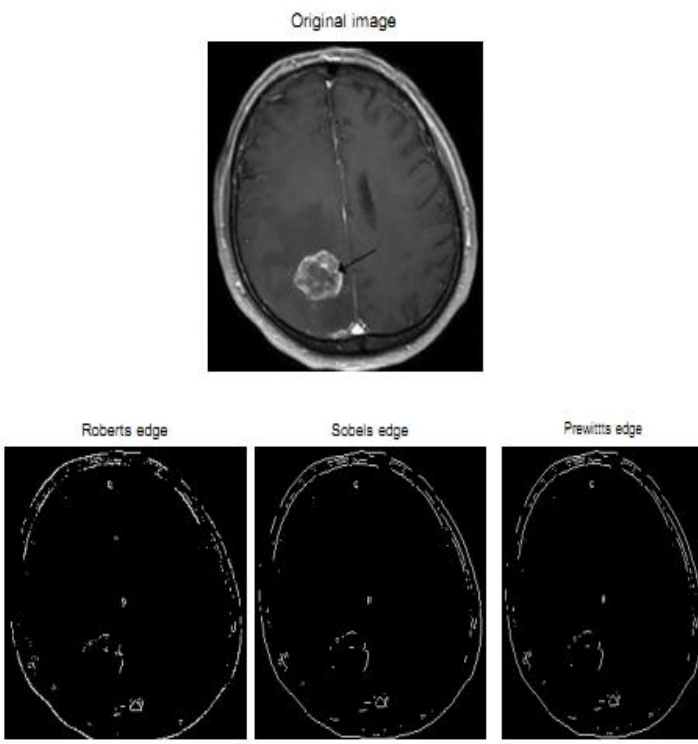

Figure 3: Results showing three different edge methods (Robert's, Sobel's, and Prewitt's)
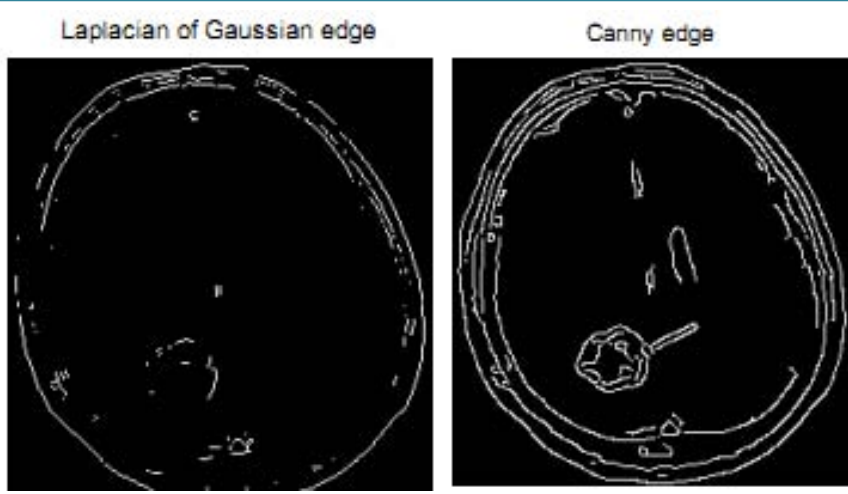

Figure 4: Results obtained using two different edge methods

\section{Advantages}

1) Second order operators give reliable results.

2)Useful in calculating the number of different objects present in the given image.

\section{Disadvantages}

1) No single operator can fit for all variety of images and the computational complexity increases with the size of operator.

2) Many a times the edges obtained are not continuous.

\section{Regional Based Segmentation}

This method works on the principle of homogeneity by considering the fact that the neighboring pixels inside a region possess similar characteristics and are dissimilar to the pixels in other regions. The objective of region based segmentation is to produce a homogeneous region which is bigger in size and results in very few regions in the image. The regions though treated as homogeneous in nature but there is provision to note any considerable changes in the characteristic of the neighboring pixels [4]. The simplest approach to segment image based on the similarity assumption is that every pixel is compared with its neighbor for similarity check (for gray level, texture, color, shape) [9]. If the result is positive, then that particular pixel is "added" to the pixel and a region is "grown" like-wise. The growing is stopped when the similarity test fails.

Region based methods are fundamentally divided as:

1) Region growing methods.

2) Region split and merge methods.

Further there are several segment approaches based on regional analysis of the image proposed by [4] Similarity measures among the neighbors, comparing the pixel to original seed, comparing the pixel to neighbor in region, comparing the pixel to the region statistics, considering multiple seeds, calculating the cumulative differences and counter examples.

\section{A.Regional Growing Methods}

Region growing method gives reliable output compared to its other counterparts. It is basically extracting a region from the image using some pre-defined criteria [8]. The simplest procedure is to compare the candidate pixel to its neighbors to check the homogeneity criteria allocated to the class

\section{Volume 6 Issue 2, February 2017}




\section{International Journal of Science and Research (IJSR) ISSN (Online): 2319-7064}

Index Copernicus Value (2015): 78.96 | Impact Factor (2015): 6.391

to which its neighbor belongs. It can be further classified as seeded region growing method (SRG) and unseeded region growing method (UsRG). The main difference between SRG and UsRG is that SRG is semiautomatic in nature whereas UsRG is fully automatic in nature [17].

\section{Seeded Region Growing Method}

It was initially proposed by Rolf Adam [10] and is found to be reliable since then. SRG has characteristics like robustness, rapidness and is also free of tuning parameters which makes it suitable for large range of images. In this approach initially the seeds are to be specified by the user. A seed is a test pixel with ideal characteristic that belongs to the region interested in and should be the part of region of interest. The choice of seed is very crucial since the overall success of the segmentation is dependent on the seed input. The seed set may have one or more members and is user's choice. For the given set of seeds, each step in the SRG adds pixels to one of the seed sets. In some approaches of SRG these seeds may be replaced by centroids of the segmented regions. The allocated pixels of same region are labeled with same value and other unallocated pixels with different value. The process is semi-automatic in nature since it depends on user for seed specification. There are several algorithms available for SRG approach. They all differ in the sense of adding pixels to a region and/or comparing the pixel with the seed. The general steps in SRG algorithm can be as follows:

1)Determine seeds to start the segmentation process.

2)Determine the criteria to grow the region. In case of multiple regions, clearly the characteristic of regions should be mentioned. So that no ambiguity exists to place the pixel in particular region.

3) The candidate pixels to include in the region it should be 8 -connected to at least one of the pixel in the region.

4)Cross-check is to be done to ensure all the pixels are tested for allocation and then label has to be given to all regions.

5)If two different regions get same label then they have to be merged.

The characteristics that are usually considered for the test of homogeneity of the region are gray level, color, texture, Shape etc.

\section{Unseeded Region Growing Method}

Unseeded region growing method is flexible, automatic segmentation approach and is based on postulate of pixel similarities within regions. UsRG does not rely on tuning parameters and is also independent of manual inputs. This approach readily incorporates the high-level knowledge of the image and is very crucial for the choice of region statistics. The incorporation of basic adaptive filtering techniques have shown some good results in practical [37]. The following are general considerations:

1)Segmentation process is initialized with region A1 containing single pixel and eventually results in several regions $\left(\mathrm{A}_{1}, \mathrm{~A}_{2}, \mathrm{~A}_{3} \ldots . . \mathrm{A}_{\mathrm{n}}\right)$ after the completion.

2)Difference measure of the test pixel with the mean value of the region statistics considered decides the pixel to be allocated or unallocated to that region.
3)If the difference value between the test pixel and the region statistics is less than certain threshold considered the pixel is allocated to that specific region, say $A_{j}$.

4)If the condition is not satisfied than the pixel considered should be allocated to new region, say $\mathrm{Ai}$ which has regional characteristic similar to the test pixel.

5)If for any test pixel if the conditions for $A_{i}$ and $A_{j}$ are not satisfied then it implies that the test pixel belongs to a new third region and so on and so forth.

\section{B. Regional Split and Merge Method}

This method is the most similar method to segment the image based on homogeneity criteria [18]. This method works on the basis of quadtrees and main objective is to distinguish the homogeneity of the image. It initially considers the entire image as one single region and then divides the image into four quadrants based on certain pre-defined criteria. It checks the quadrants for the same defined criteria and divides it further into four quadrants if the test result is negative and the process continues till the criteria is satisfied or no further division is possible. The figure given below illustrates the process and also the algorithm is given below:

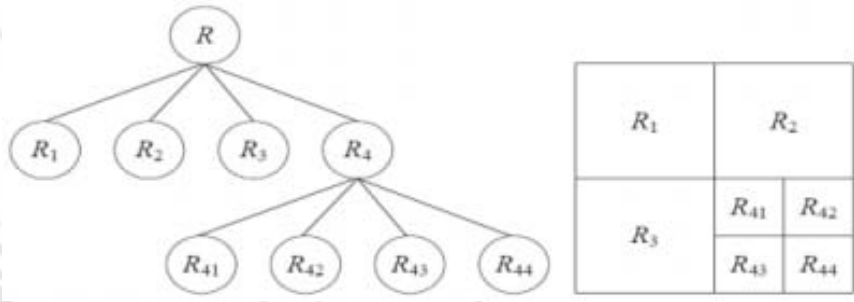

Algorithm:

Let $\mathrm{R}$ represent the entire image region and let $\mathrm{P}$ be any predicate.

If $\mathrm{P}(\mathrm{R})==$ False

Divide the image into quadrants

If $P$ if false for any subquadrant

Subdivide that quadrant into subquadrant...

Stop dividing when $\mathrm{P}$ is true.

Merge the regions $R_{j} \& R_{k} \quad(j \neq k ; j=1,2,3 \ldots n$, $\mathrm{k}=1,2,3 \ldots \mathrm{n})$

$$
\text { if } P\left(R_{j} U R_{k}\right)==\text { true }
$$
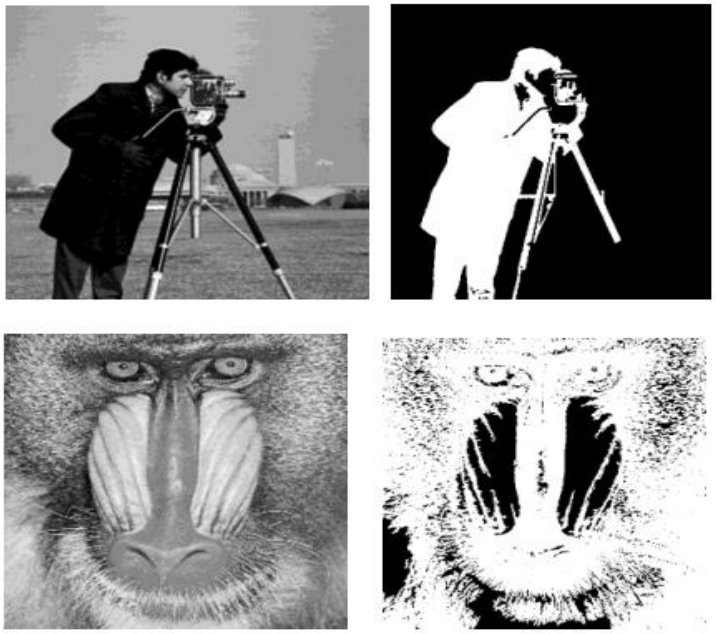

Figure 5: Results of regional split and merge method

\section{Volume 6 Issue 2, February 2017




\section{International Journal of Science and Research (IJSR) \\ ISSN (Online): 2319-7064}

Index Copernicus Value (2015): 78.96 | Impact Factor (2015): 6.391

\section{Advantages}

1) Gives superior results compared to other segmentation methods.

2) Provides flexibility to choose between interactive and automatic techniques to segment the given image.

3) Since it flows from inner point to outer region more likely to get clear boundary for the objects.

4) If proper seed is selected it gives very accurate result than any other methods.

\section{Disadvantages}

1) Formulation of stopping rule for segmentation is a tedious task [20].

2) SRG can result in scan-order dependencies and can have significant impacts on small regions.

3) A good segmentation result depends on a set of correct choice for the seeds and can lead to erroneous segmentation results if user specifies a noisy seed.

4) The seed selection process in itself requires manual interventions, and is error-prone.

\section{Discussion}

Image segmentation is a process of dividing an image into its constituent homogeneous regions to extract data from the attributes of the image. As a result, a good segmentation should result in regions in which the image elements should have uniform properties in terms of brightness, color or texture etc. Though the image is to be portioned into regions, the considerable changes within the regions should be observable visually. The measurement of quality of segmentation is that the elements of the same region should be similar and should have clear difference between elements of the other regions. The segmentation process can be divided into various category based on the parameter selected for segmentation like pixel intensity, homogeneity, discontinuity, cluster data, topology etc. Each approach has its own advantages and disadvantages. The result obtained using one approach may not be the same as compared with other approach. Methods that are specialized to particular applications can often achieve better performance and the selection of an appropriate approach to a segmentation problem can be a difficult problem.

Basically the segmentation can be semi-interactive or fully automatic. The algorithms developed for segmentation lies in either of this category. With the major difficulty of illposed nature of segmentation it is hard to obtain single answer for segmentation of given image as the interpretation varies from individual approaches. So a single approach to segment all variety of images may be practical unachievable. The prior knowledge on the image can give better results and gives user the choice to decide proper method to segment the image.

\section{Acknowledgement}

This work was supported by the China Scholarship Council (CSC), under student number 07M2014006L, through the Department of Signal and Information Processing, School of Electronic Engineering, Tianjin University of Technology and Education, 1310 Dagu South Road, Hexi District, Tianjin 300222, P. R. China.

\section{References}

[1] Ajala Funmilola A, Oke O.A, Adedeji T.O, Alade O.M, Adewusi E.A. "Fuzzy k-c-means Clustering Algorithm for Medical Image Segmentation" Journal of Information Engineering and Applications ISSN 2224-5782 Vol 2, No.6, 2012

[2] Alamgir Nyma, Myeongsu Kang, Yung-Keun Kwon, Cheol-Hong Kim, and Jong-Myon Kim "A Hybrid Technique forMedical Image Segmentation" Article ID 830252,Journal of Biomedicine and Biotechnology Hindawi Publishing Corporation Volume 2012.

[3] Ravi S and A M Khan, "Operators Used in Edge Detection: A Case Study", International Journal of Applied Engineering Research, ISSN 0973-4562 vol. 7 No 11, 2012.

[4] Bryan S. Morse, Lecture 18: Segmentation (Region Based), Brigham Young University, 1998

[5] Dr. (Mrs.) G.Padmavathi, Dr.(Mrs.) P.Subashini and Mrs.A.Sumi Empirical Evaluation of Suitable Segmentation Algorithms for IR Images", IJCSI International Journal of Computer Science Issues, Vol. 7, Issue 4, No 2, July 2010.

[6] Dr.S.V.Kasmir Raja, A.Shaik Abdul Khadir And Dr.S.S.Riaz Ahamed, "MOVING TOWARD REGION-BASED IMAGE SEGMENTATION TECHNIQUES: A STUDY", Journal of Theoretical and Applied Information Technology.

[7] Nobuyuki Otsu, "A Threshold Selection Method From Gray-Level Histograms", IEEE Transactions on Systems, Man, And Cybernetics, Vol. Smc-9, No. 1, January 1979

[8] Dzung L. Pham, Chenyang $\mathrm{Xu}$, and Jerry L. Prince, "CURRENTMETHODS INMEDICAL IMAGE SEGMENTATION”, Annu. Rev. Biomed. Eng. 2000. Vol. 02 page no. 315-37.

[9] H.S.Prasantha, Dr.Shashidhara.H.L, Dr.K.N.B.Murthy and Madhavi Lata.G, "MEDICAL IMAGE SEGMENTATION", (IJCSE) International Journal on Computer Science and Engineering Vol. 02, No. 04, 2010.

[10]Rolf Adams and Leanne Bischof, "Seeded Region Growing", IEEE Transactions on Pattern Analysis and Machine Intelligence, Vol.16, No. 6, June 1994.

[11]N. Senthilkumaran and R. Rajesh, "Edge Detection Techniques for Image Segmentation - A Survey of Soft Computing Approaches", International Journal of Recent Trends in Engineering, Vol. 1, No. 2, May 2009.

[12] Nikhil R Pal and Sankar K Pal, "A Review on Image Segmentation Techniques", Pattern Recognition, Vol 26, No. 29, 1993.

[13]X. Munoz, J. Freixenet, X. Cuf_1, J. Mart, "Strategies for image segmentation combining region and boundary information", Pattern Recognition Letters 24, page no 375-392, 2003.

[14] Tranos Zuva, Oludayo O. Olugbara, Sunday O. Ojo and Seleman M. Ngwira "Image Segmentation, Available Techniques, Developments and Open Issues",

\section{Volume 6 Issue 2, February 2017}


Canadian Journal on Image Processing and Computer Vision Vol. 2, No. 3, March 2011.

[15] WANG Hongzhi and DONG Ying, "An Improved Image Segmentation Algorithm Based on Otsu Method", International Symposium on Photoelectronic Detection and Imaging 2007: Related Technologies and Applications, Vol. 6625, 2008.

[16] Yuri Y. Boykov and Marie-Pierre Jolly, "Interactive Graph Cuts For Optimal Boundary \& Region Segmentation Of Objects In N-D Images", Proceedings Of "Internation Conference On Computer Vision", Vancouver, Canada, Vol.I, P.105, July 2001.

[17]Zheng Lin, Jesse Jin and Hugues Talbot, "Unseeded region growing for 3D image segmentation".

[18]Bo Peng, Lei Zhang, and David Zhang, "A Survey of Graph Theoretical Approaches to Image Segmentation".

[19]R.C. Gonzalez and R.E. Woods, "Digital Image Processing", third edition, PHI publication, 2008.

[20] S. Nagabhushana, "Computer Vision and Image Processing”, New age International Publishers, 2005.

\section{Author Profile}

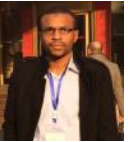

Mohammed Jajere Adamu received the B.Eng. degree in Computer Engineering from University of Maiduguri, Nigeria in the year 2012. He is currently a Masters Research Student in the Institute of Antenna and Microwave Techniques, Tianjin University of Technology and Education, Tianjin, P. R. China. He holds a number of IT certifications among which are CCNA, CCNP R\&S, MCP, and MCSA. His research interest includes; Antenna Design, Modern Wireless Network Design, Network Management and Network Security, and Image Processing.

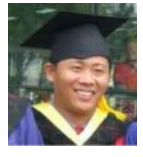

Xuewen Ding received his B.Eng. and Ph.D. degrees in signal and information processing from Tianjin University, Tianjin, in 2005 and 2008 respectively. He is currently an Associate Professor in the School of Electronic Engineering, Tianjin University of Technology and Education. He has published over 10 papers in video and image processing and applied for two patents. His current area of research include digital image processing, video processing and transmission, multimedia search, and machine vision. He has acted as a primary role in multi-projects such as National Nature Science Foundation of China, Key Nature Science Foundation of Tianjin, and Tianjin Science \& Technology Fund Planning Project. 